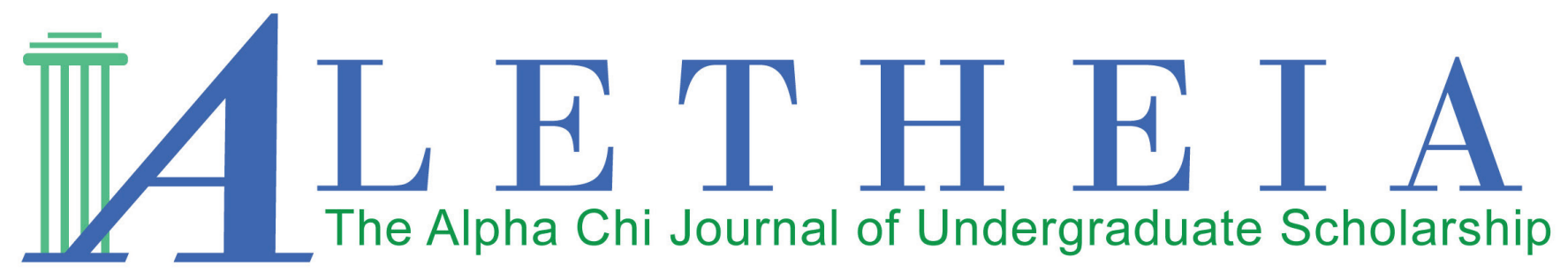

Volume 2 | Issue 1 | 2017

\title{
An Analysis of Empirical Validity of Alfred Adler's Theory of Birth Order
}

\author{
Kathleen E. Marano \\ Caldwell University \\ New Jersey Alpha Chapter
}

Vol. 2(1), 2017

Article Title: An Analysis of Empirical Validity of Alfred Adler's Theory of Birth Order

DOI: $10.21081 / \mathrm{AX} 0082$

ISSN: 2381-800X

Key Words: Alfred Adler, birth order, empirical validity, personality formation

This work is licensed under a Creative Commons Attribution 4.0 International License.

Author contact information is available from the Editor at editor@alphachihonor.org.

\section{Aletheia-The Alpha Chi Journal of Undergraduate Scholarship}

- This publication is an online, peer-reviewed, interdisciplinary undergraduate journal, whose mission is to promote high quality research and scholarship among undergraduates by showcasing exemplary work.

- Submissions can be in any basic or applied field of study, including the physical and life sciences, the social sciences, the humanities, education, engineering, and the arts.

- Publication in Aletheia will recognize students who excel academically and foster mentor/mentee relationships between faculty and students.

- In keeping with the strong tradition of student involvement in all levels of Alpha Chi, the journal will also provide a forum for students to become actively involved in the writing, peer review, and publication process.

- More information and instructions for authors is available under the publications tab at www.AlphaChiHonor.org. Questions to the editor may be directed to editor@alphachihonor.org.

\footnotetext{
Alpha Chi is a national college honor society that admits students from all academic disciplines, with membership limited to the top 10 percent of an institution's juniors, seniors, and graduate students. Invitation to membership comes only through an institutional chapter. A college seeking a chapter must grant baccalaureate degrees and be regionally accredited. Some 300 chapters, located in almost every state, induct approximately 12,000 members annually. Alpha Chi members have been "making scholarship effective for good" since 1922.
} 


\title{
An Analysis of Empirical Validity of Alfred Adler's Theory of Birth Order
}

\author{
Kathleen E. Marano \\ Caldwell University \\ New Jersey Alpha Chapter
}

\begin{abstract}
There are many theories that attempt to explain the formation of personality. This paper examines Alfred Adler's theory of birth order and draws conclusions about its empirical validity. It describes how Adler's own life directly influenced his work and the theory itself, including the five distinct personality types that would develop as a result of birth order position. The research that has been conducted on the topic is then presented, focusing on both the overall personality types and specific traits, as well as research methodology and possible factors that could alter birth order effects. The paper concludes with an analysis of the research in terms of its flaws, limitations, and comprehensiveness in order to determine if there is empirical support for the theory. Suggestions for future research are then presented.
\end{abstract}

Key words: Alfred Adler, birth order, empirical validity, personality formation

One of the most studied and interesting areas in the field of psychology is the formation of personality and the various factors that impact it. Over the course of many years, multiple theories have been formulated that attempt to explain the phenomenon behind the development of each individual's characteristics. Many well-known psychologists, as well as other prominent figures, have contributed their thoughts on the subject, resulting in a multitude of differing opinions and theories (Ryckman, 2013). One of the most compelling of these theories is that developed by Alfred Adler. Born in 1870 and initially trained as a medical doctor, Adler was a psychotherapist and the founder of Adlerian Psychology and Individual Psychology (Ryckman, 2013). While his work in the field of psychology is quite comprehensive, it is his theory of birth order that is most relevant to the development of specific personality traits. Adler believed that the experiences each individual underwent as a result of his or her order of birth helped shape their characteristics (Ansbacher \& Ansbacher, 1956). Adler also wrote about the traits expected for each of the ranks of birth order. A great deal of subsequent research has been conducted in order to determine the heuristic value of his work. Because all individuals can fit into one of Adler's categories of birth order, many researchers are interested in testing the accuracy of his predicted characteristics for each rank. This paper will explore Adler's theory of birth order and the various studies that have been conducted in order to determine the empirical validity of his work.

\section{Adler's Life}

Alfred Adler was born in Vienna in 1870 to a middle-class family with seven children. He was the third child; the first child was a boy and the second a girl. Throughout his childhood, Adler suffered from rickets, was very weak, and fell ill often, which resulted in his 
parents pampering him (Ryckman, 2013). His health problems led to a feeling of inferiority, as well as a sibling rivalry with his older brother, Sigmund. One of his writings includes a description of a day his family was at the beach and he became jealous of Sigmund's health. He wrote, "He could run, jump, and move about quite effortlessly, while for me, movement of any sort was a strain and an effort" (Eckstein \& Kaufman, 2012, p. 63). Adler also felt that his brother was the favored one in the family and grew up being compared to him (Eckstein et al., 2010). Therefore, Sigmund felt very inferior to his eldest sibling, a childhood experience that may have contributed to his birth order theory.

According to Ryckman (2013), as Adler grew up, he attended Vienna Medical School and ultimately became a physician and psychotherapist. He established his own practice in Vienna and treated mainly lower-middle-class patients. In 1899, he began to correspond with Sigmund Freud about one of his patients and was later asked to become part of Freud's weekly discussion group. Freud even recommended him as his successor as president of the Vienna Psychoanalytic Society. However, Adler never developed a close relationship with Freud and often publicly disagreed with his work, leading to his resignation from the society in 1911. Adler named his own association the Society for Free Psycho-Analytic Research in order to show his displeasure with Freud's "dictatorial ways" (Ryckman, 2013, p. 78). It is interesting that Adler grew up being compared to his brother Sigmund and later spent much of his career under the shadow of Sigmund Freud. Therefore, his feelings of in feriority and the constant comparisons he experienced may have impacted the development of his work in the field of psychology.

\section{Adler's Theory}

Sigmund Freud is perhaps the most well-known contributor to the field of psychology. His influence was so great that many of those who came after him found it hard to escape his shadow. As previously mentioned, both Adler himself and his work were often compared to Freud and his work, although Adler openly disagreed with many of Freud's theories and ultimately created his own school of thought. While Adler initially named his association the Society for Free Psycho-Analytic Research to show his dissent from other psychologists, he later renamed it the Society for Individual Psychology (Eckstein \& Kaufman, 2012).
Adler and Freud were similar in several ways, but they also disagreed in multiple areas. Both men were initially trained as medical doctors, with Adler attending medical school to become a physician and Freud training in neurology (Ryckman, 2013). However, they used their medical backgrounds in different ways to advance the field of psychology. According to Ryckman (2013), while Freud felt that human motivation was inborn and focused on the nature aspect of development, Adler believed development was primarily social and focused on the nurture aspect. Freud emphasized human similarity, whereas Adler emphasized human uniqueness. Their final main point of contention was over whether motivation was conscious or unconscious, with Adler believing the former and Freud focusing on the latter (Ryckman, 2013). Therefore, while Adler is often seen in the same light as Freud, his theory differs greatly in several key areas.

Adler believed that individuals must be studied in terms of their whole personality, which is reflected in his decision to call his school of thought Individual Psychology. However, he believed that individuals could only be understood in terms of their interactions with other people (Ryckman, 2013). Therefore, Individual Psychology focuses on understanding "the experiences and behavior of each person as an organized entity" (Ryckman, 2013, p.78). He believed that all human behavior is driven by goals and the ultimate desire to be superior, which is motivated by feelings of inferiority. All people have some feelings of inferiority, which can be either organ related, social, or psychological (Ansbacher \& Ansbacher, 1956). Thus, Adler's theory focuses on the social components of human behavior and humans' striving for improvement.

While his writings are very comprehensive, the part most relevant to the formation of personality is his extensive work on birth order and how it relates to the development of certain traits. Adler believed that one's rank within the family would impact the individual's experiences, thereby altering the way that individual's personality develops. The traits expected for each child are not dependent on their actual order of birth, but rather on the social interactions they experience as a result of that factor. Adler wrote, "It is not, of course, the child's number in the order of successive births which influences his character, but the situation into which he is born and the way in which he interprets it" (Eckstein et al., 2010, p. 409). Thus, an individual's order of birth does not necessarily bestow certain traits to him or her, but 
it does impact the situations and experiences that will ultimately shape his or her personality.

Adler also emphasized that there are many aspects of birth order other than the number of successive births that play a role in the development of certain characteristics. According to Ansbacher and Ansbacher (1956), he stated that an individual's own perception of his or her birth order is more important than the actual order. This psychological perception of birth order can be impacted by multiple factors. In families with children with disabilities, the perceived birth order of each child may be altered. For example, if the eldest child is disabled, the second born child may take on the role of the first born and therefore develop the characteristics of that rank. Birth order can also be impacted by the death of a child. Adler wrote about his theory in the 1920s and 1930s, a time when the death of a child was not uncommon. Hence, a child's actual birth order is susceptible to change. If a family is very large and there is a significant age difference among the groups of children, the eldest child of a later group may develop in the way of a first child, despite not actually being born first. Differences such as these may also occur in the case of twins (Ansbacher \& Ansbacher, 1956). According to Ryckman (2013), gender can also alter how people view themselves within their families. For example, if a girl is the first born and the second born child is a boy, in a patriarchal society the boy may take on the role of the first born child. Birth order impacts can also be changed due to gender if there is a large number of all same-sex children with the exception of one. A single male child in a family of girls is more likely to experience social difficulty, while a female in a family of males will likely either develop very feminine or very masculine qualities (Ryckman, 2013). Therefore, many factors can impact how an individual interprets their experiences and perceives their birth order, causing their perception to play a more significant role than merely the individual's place in a succession of births.

In addition to individuals' experiences as a result of birth order shaping their personalities, Adler also cited a process called sibling de-identification as a reason for the development of specific personality types for each child. According to Eckstein and Kaufman (2012), because the majority of children have at least one sibling, they often work to define themselves differently from one another, either consciously or unconsciously, in order to have their own identities and earn their own share of parental support and attention. Through this process of de-identification, children are able to attain their own attributes, behaviors, and unique tendencies within their families. They also use sibling identification and modeling among each other. Older siblings often serve as models for younger children and help care for them, which can result in younger siblings imitating their behavior (Eckstein \& Kaufman, 2012). Therefore, the interplay of perceived birth order, de-identification, and modeling and imitation often result in the creation of specific personality types for each rank of birth.

\section{The First Born Child}

Adler described five distinct categories of birth order which can be applied to all individuals, and assigned certain traits to those who fit into each group. The first of those categories is the oldest child, who typically receives a great deal of attention before the birth of subsequent siblings. Because there are no other siblings to compete with, first children receive their parents' full affection during the beginning of their lives. However, after the birth of the second child, the first born takes on the role of the "dethroned monarch," forced to share parental attention with the new sibling (Ryckman, 2013, p. 84). The amount of time between births can also have an impact on how children cope with the birth of a new sibling. If it is three or more years, a routine of life has already been established and is responded to accordingly, whereas a lesser time interval means that the individual will not be able to understand the change with words or concepts (Ansbacher \& Ansbacher, 1956). Therefore, it is more difficult for such children to understand what has happened.

The oldest child may feel resentment and hostility toward younger siblings because the younger siblings "dethroned" the oldest child from his or her previous position in the family. This is more likely to occur if parents do not properly prepare children for the birth of a sibling. If a child is not sufficiently prepared, they are more likely to experience neuroses, but with proper handling, the oldest child may take on the role of another parent (Ryckman, 2013). Therefore, some oldest children will be protective, supportive, and nurturing of younger siblings. Because they often act as a third parental figure, first born children may develop organizational talents. However, they may develop a desire to protect others, which results in the need to keep others 
dependent on them (Ansbacher \& Ansbacher, 1956). According to Ryckman (2013), the oldest child best understands the importance of power and authority because they have had to undergo the loss of it. They will be more supportive of and dependent on authorities, as well as politically conservative and conforming (Ryckman, 2013). They may also prefer order, structure, and adherence to norms and rules (Stewart, 2012). Oldest children may also be past-oriented because their focus is on the time when they were the center of attention (Ansbacher \& Ansbacher, 1956). Therefore, eldest children may be protective and supportive, or neurotic and insecure, depending on how well the birth of a new sibling is handled.

\section{The Second Born Child}

The next classification of birth order described by Adler is that of the second child, which is defined as someone who is born second, but will ultimately have younger siblings (Ryckman, 2013). According to Ansbacher and Ansbacher (1956), this child must share attention with another sibling from birth and is therefore more likely to be cooperative than the first born. They tend to be very competitive as they are constantly striving to keep up with their older sibling. This trend often continues in their careers as they work harder than others in order to be the best (Ansbacher \& Ansbacher, 1956). However, they may set unrealistically high goals for themselves, which will essentially ensure their ultimate failure. This can result in neurosis later in life because their expectations for themselves can never be met. Later in life, the second child is more likely to resist authority and believe that there is no power that cannot be overthrown (Ryckman, 2013).

\section{The Youngest Child}

The third category of birth order is the youngest child, who is the last born person in the family. Since new siblings will not dethrone the youngest child, they are often the pampered babies of the family. They may hold the majority of the family's attention, which can result in an excessive dependency on others for support and protection (Ryckman, 2013). Since the youngest child has at least one older sibling, they have many opportunities to compete with others. Due to the increased attention they receive, they may develop in an extraordinary manner and excel in their endeavors, often surpassing their competitors (Ansbacher \& Ansbacher, 1956). They are given many opportunities to do well, and success in such situations can catapult them into further achievement, earning them recognition within their families. However, since they are the youngest, their families often indulge them. This may result in these individuals seeking easy solutions to problems and learning how to coax or charm others into doing what they ask. Due to this ability, they are often seen as the most popular out of the different ranks of birth order (Ansbacher \& Ansbacher, 1956).

According to Stewart (2012), Adler also wrote that some youngest born children may become easily discouraged in their tasks and not establish socially useful roles with their siblings. They may then use their failures and mistakes as a way to find significance among their family members. While Adler wrote that some youngest children are able to overcome all competitors and be very successful due to the added support from their families, he also theorized that they are highly likely to be problem children if they are spoiled. In addition, Adler believed that youngest children were the most likely and most suited to become counselors. According to Ansbacher and Ansbacher (1956), such children will never gain independence and will often not identify a single ambition because they want to excel in all things. In some cases, they may also suffer from extreme inferiority because they feel younger, weaker, and less experienced than others in society (Ansbacher \& Ansbacher, 1956). Thus, there is significant variability among the characteristics of the youngest child, ranging from a competitive, successful individual to a problem child who is unable to complete tasks without assistance.

\section{The Only Child}

The fourth classification described by Adler is the only child, which is defined as a child with no siblings. Since there are no sibling rivals, these children have their parents' full attention throughout their upbringing and are often pampered (Ryckman, 2013). According to Stewart (2012), this can result in feelings of entitlement and dependence even when outside the family. However, some only children may feel smothered by the attention of their families and seek independence and autonomy (Stewart, 2012). According to Ansbacher and Ansbacher (1956), the lack of siblings can also lead to problems for 
only children. The feelings of competition may be directed toward their fathers, while their mothers pamper them. This can lead to a mother complex, in which the child wants his or her mother's full attention at all times and wants to remove the father from the family picture (Ansbacher \& Ansbacher, 1956). Therefore, the vast amount of attention given to only children can result in significant difficulties.

Other problems can arise for only children because of their parents. According to Ansbacher and Ansbacher (1956), parents of only children who are expected to have more children are often pessimistic. The parents may have feared they would be unable to cope with the economic issues they would face if they had additional offspring. The child grows up in an anxiety-filled atmosphere and suffers as a result (Ansbacher \& Ansbacher, 1956). Another potential scenario for only children is that their parents may not have wanted children. If this is the case, only children may face active rejection or neglect, which could have a lifelong, damaging impact (Ryckman, 2013, p. 85). Therefore, only children may suffer from negative personality traits for a variety of reasons due to their lack of siblings. Adler also noted that children in larger families who are separated by many years may develop some of the characteristics of only children (Ansbacher \& Ansbacher, 1956).

\section{The Middle Child}

The final classification of birth order character types is the middle child, which is defined as a child who has at least one older sibling and at least one younger sibling (Ryckman, 2013). According to Stewart (2012), because they lack the "primacy of the first child and the attention-garnering recency of the youngest child, persons in the middle role may feel like they were squeezed out of their families" (p.78). Middle children may perceive themselves in a negative light because they lack the distinctions that come along with being first or last born. They may think they have no attributes that make them special and worthy of the attention of their families. Therefore, some middle children may feel less loved than their siblings and feel rejected. Individuals who are able to successfully overcome these feelings "may emerge with well-developed interpersonal skills and an enhanced sense of self-esteem" (Stewart, 2012, p. 78). Thus, middle children may be likely to experience difficulties due to their order of birth, but they may also be capable of overcoming them. Each of the five classifications comes with a predicted personality type that may develop as a result of an individual's order of birth.

\section{Research Methodology}

Adler's five classifications of birth order can be applied to all individuals; and if Adler's theory is accurate, birth order could play a significant role in the development of personality. For this reason, many psychologists and researchers have invested time in conducting studies to test the empirical validity of the theory of birth order. Between 1990 and 2010, approximately 670 publications contained "birth order" as a major subject descriptor (Stewart, 2012). These studies vary widely in scope and focus, although those discussed in this paper focus specifically on personality traits that may develop as a result of birth order.

The research conducted on Adler's theory of birth order typically falls into one of two categories. Researchers may choose to base their studies on either actual or psychological birth order. According to Stewart (2012), actual birth order is defined as "the numerical rank order in which siblings were born into or entered the family of origin" (p. 76). Thus, one's actual birth order refers to simply where he or she falls within the successive number of births in the family. Stewart (2012) defines psychological birth order as one's perceived position within their family, including the situation into which the person was born and his or her interpretation of it. Therefore, the psychological position may differ from one's actual birth order. As previously discussed, the gender of siblings, the death of a sibling, or the disability of a sibling may impact one's psychological birth order position. Thus, individuals may be the second child, but consider themselves to have the role of a first born child.

In all of Adler's writings, he emphasized that psychological birth order is much more important than actual birth order. This is because the child's self-perceived position within the family impacts the ways the child approaches the "tasks of completion and belonging" (Stewart, 2012, p. 77). Adler felt that it is not the events that actually occur, but rather the individual's own interpretation of such events that impacts his or her development. However, while Adler wrote that psychological birth order is more important, the majority of studies focus on actual birth order. This is because it can be difficult to reliably identify one's perceived birth order and 
it can be more time-consuming to conduct the lifestyle interviews necessary for researching psychological birth order (Stewart, 2012). Because there is such a distinction between the types of birth order and because most research focuses on actual birth order, it is possible that many of the studies are flawed for this reason.

\section{Research on Birth Order Positions}

A great deal of the research on Adler's birth order theory has centered on the classifications of birth order positions within the family. Since his work included descriptions of the five distinct personality types that could develop, many people chose to analyze whether or not individuals actually possessed such traits. Because the research in this area is so extensive, Eckstein et al. (2010) published a journal article that summarized and reviewed 200 birth order studies. They searched various databases for statistically significant research on birth order and then compiled the results into one cohesive article, including studies and literature from between 1960 and 2010. Eckstein was also involved in a 2000 study in which he reported on 151 research articles in order to compile birth order characteristics for each position. Eckstein's work from both 2000 and 2010 can be examined in order to determine how the research has changed over time and whether the findings have remained consistent with Adler's original theory.

While Adler wrote about five possible birth order positions, the studies analyzed by Eckstein (2000) and Eckstein et al. (2010) focus on only four. The researchers chose to combine Adler's separate classifications of the second born and the middle child into the category of the middle child. They defined the first born child as "the first child born in a family with subsequent siblings" (Eckstein et al., 2010, p. 417). Only children refer to first born children who do not have subsequent siblings. Middle children are defined as "all children born between the first born child and the youngest child" (Eckstein et al., 2010, p. 418). The final category is youngest children, who are the "last children born of families with two or more children" (Eckstein et al., 2010, p. 418). Eckstein (2000) and Eckstein et al. (2010) used these four classifications to compile the various studies in order to determine the characteristics most commonly found in individuals of each position.

According to Eckstein (2000), only children have the greatest need for achievement, are the highest achievers of all birth order ranks except oldest children, are the most likely to attend college, and are the most likely to manifest behavior problems (Eckstein, 2000). These findings remained true ten years later when Eckstein conducted his second examination into the personality types that may develop as a result of birth order. According to Eckstein et al. (2010), only children are ranked highest in achievements and intelligence with the exception of oldest children, have the highest need for achievement, are more likely to go to college, have more behavior problems, have the lowest need for affiliation, are more selfish, and have a significant percentage of psychiatric disorders. These results are also consistent with Adler's initial theory. He proposed that such children may be successful as result of the amount of parental support they receive, which is evident in their high levels of achievement (Ryckman, 2013). However, the potential problems that may occur due to their parents and lack of siblings are also evident in the analyses of birth order studies since only children may exhibit problem behavior and psychiatric disorders. Therefore, the personality traits that Adler believed may develop in only children are supported by the empirical research conducted many years later by Eckstein (2000) and Eckstein et al. (2010).

Eckstein (2000) described the personality traits most often found among oldest children. They tend to be the highest achieving, have the greatest academic success with fewest academic problems, have the highest motivation and need for achievement, are overrepresented among learned groups such as college students and faculty, and are the most affinitive when under stress (Eckstein, 2000). Eckstein et al. (2010) found the same results and also expanded on these personality traits. Their results showed that oldest children are also the most likely to be leaders, are the most influenced by authority, and are more likely to be responsible and conscientious. Overall, oldest children tend to be the highest achieving of all possible birth order positions and tend to be more active in holding political positions. This is especially consistent with Adler's theory and the traits he attributed to first born children. He also believed they might have more organization skills, which is reflected in their increased likelihood for being responsible. Adler also wrote about the firstborn's appreciation for authority and their high success levels, which is evident in their overrepresentation among politicians and leaders, as well as their high motivation and achievement (Ryck- 
man, 2013). Thus, there is empirical support for the theory of birth order in the research on the characteristics of oldest children.

The next position of birth order in Eckstein's research is that of the middle child. According to Eckstein (2000), middle children have the fewest acting-out problems, are the most sociable, and have the greatest feelings of not belonging (Eckstein, 2000). Eckstein et al. (2010) found similar results in their analysis of birth order research. They learned that middle children often have feelings of not belonging, are sociable, act out the least, have success in team sports, relate well to older and younger people, and compete in different areas than the oldest.

These results make sense in relation to the birth order position of middle children due to the presence of siblings. Because they were always in the company of older and younger siblings, it is logical that they would relate well to those of different ages, be the most sociable, and perform well in sports. By growing up in the presence of siblings, middle children are forced to learn how to cooperate with others. The findings of the hundreds of studies also show support for Adler's theory because they demonstrate that middle children often feel they do not belong. His work focused on the idea that they may feel they hold no special place in their families and are being squeezed out of them because of their perceived lack of attributes (Ryckman, 2013). The findings of the studies were consistent with Adler's work, showing that an analysis of birth order research yields empirical support for his theory.

The final birth order position is that of the youngest child and the character traits that are most often attributed to those who fall into that category. According to Eckstein (2000), youngest children make up the greatest percentage of those with psychiatric disorders if they are from a small family, have the greatest tendency toward abusing alcohol, and are the most empathetic (Eckstein, 2000). These findings are consistent with Adler's theory because he felt that youngest children often sought easy solutions to their problems, which can be shown in their tendency toward alcohol. He also felt that they were the most likely to pursue careers as counselors, which is supported through their increased likelihood for empathy (Ansbacher \& Ansbacher, 1956). Those who possess empathy are more likely to achieve success in such careers.
Eckstein et al. (2012) greatly expanded on the findings from ten years earlier. While they also found that youngest children show empathy, are more likely to become alcoholics, and show an overrepresentation of psychiatric disorders, they also discovered a great deal of other characteristics that youngest children tend to exhibit. According to their article, youngest children have the highest social interest and agreeableness, are the most rebellious, are more artistic and less scientific, are perceived as spoiled, and are the most popular. Just as Adler's work was supported by empirical research in the other possible birth order positions, his theory regarding the personality traits expected for youngest children is also corroborated. Adler's theory of de-identification is upheld through the differences between oldest and youngest children. Oldest children are more involved in the sciences and politics, whereas youngest children are more artistic. Adler wrote that children often express an interest in fields that are different from their siblings' in order to be viewed as unique. Therefore, it is logical that youngest children would be inclined to excel in opposite areas. Adler's hypothesis that youngest children are often pampered is reflected in the finding that they tend to be spoiled. This phenomenon can also result in their ability to charm others, which is supported by their social interest and popularity. Thus, Adler's comprehensive theory about the possible personalities that may develop as a result of birth order positions is supported through empirical research. Studies show that his ideas are upheld across all possible birth ranks.

\section{Research on Specific Traits}

While many psychologists chose to perform research studies focusing on the possible birth order positions and the distinct personalities that may form as a result, others focused on specific traits in relation to birth order. Eckstein and Kaufman (2012) examined many of these studies in order to create a comprehensive overview. One of the topics they covered was teaching interactions between siblings and the concept of learning. According to Adler's theory, many of the social interactions that occur during childhood are between siblings. Older siblings may act as teachers and role models for younger children. In their article, Eckstein and Kaufman (2012) present a study by Recchia et al. (2009) in which researchers decided to examine this concept further by 
comparing the teaching strategies used with first born and second children.

Recchia et al. (2009) studied the teaching interactions between 72 sibling dyads in order to study the teachers' speech, specificity of instructions, learner involvement, and how learner errors were corrected. The purpose of the study was to learn more about how teaching strategies were impacted by age gaps between siblings and teacher birth order (Eckstein \& Kaufman, 2012). They found that learner involvement was different depending on which sibling was in the role of teacher. Older siblings tended to allow less active participation of younger siblings in the error correction process, whereas younger siblings acting as teachers fostered a more collaborative process (Recchia et al., 2009). This is consistent with Adler's work because it shows that first born children may view their younger siblings as less competent. Thus, they allow them to participate less since they doubt they will able to understand the material as well. More collaboration occurs when younger children act as teachers because the hierarchical interactions are likely to come into play. First born children may feel that they are capable of learning whatever their less experienced siblings are teaching and therefore ask more questions to ensure that they understand. Therefore, the results of the study showed that the perceived ability of the learner relative to the teacher is very important for teaching relationships between siblings (Eckstein \& Kaufman, 2012).

Eckstein and Kaufman (2012) also covered the topic of occupation in relation to birth order. They found that people's perceptions of birth order carry over to how they view those involved in different careers. Participants in studies believed that first born children have an increased likelihood to hold more prestigious jobs, whereas last born children were seen as pursuing less conservative careers. For example, participants thought that first born children were more likely to become astronauts, whereas last born individuals were more likely to become musicians (Eckstein \& Kaufman, 2012). Another study examined focused on the careers most often held by first born children (Eckstein \& Kaufman, 2012). They were overrepresented among U.S. presidents, leaders of countries all over the world, members of the U.S. Congress, and state governors (Eckstein \& Kaufman, 2012). This representation may be the result of parenting techniques in which parents often favor their first born children as a result of living in patriarchal societies. It is also possible that parents put more time and effort into their first child since they must divide their attention more as subsequent siblings are born. Older siblings may also take on a role of authority with their younger siblings, which may predispose them for careers that require them to take on a leadership position. The finding that first born children tend to pursue political careers more often than those of other birth order ranks is consistent with Adler's theory that first born children are more likely to favor authority figures.

Eckstein and Kaufman (2012) also discussed intelligence and the possible ways it can be impacted by birth order. Many people believe that first born children have the highest IQ scores and that the scores of each successive sibling are lower than those of the oldest child. This may be the result of a lack of parental attention because younger siblings are forced to share parental support, whereas the first born child initially receives their full attention. The confluence model was developed in order to account for intelligence at various ages. It reflects the concept that as siblings age, their mental maturities flow together over time and influence each other. According to the confluence model, the impact of birth order on intelligence is not found until after age eleven. After earliest born children reach eleven years of age, there is a distinct increase in their IQ scores in relation to subsequent siblings. Therefore, there is some empirical evidence to support the idea that first born children tend to be more intelligent, although Eckstein and Kaufman (2012) warn that there is still a need for more studies on the topic.

\section{Factors Impacting Birth Order Effects}

As previously discussed, research studies have been conducted on the various personalities that may develop as a result of birth order effects, as well as the presence of specific traits in siblings. The results of these studies have shown support for Adler's theory and are consistent with his thoughts on the development of personality. Some other researchers have chosen to focus on Adler's work on the factors that may limit or impact the effects of birth order. The most significant factors that have been found to alter birth order effects are the genders of siblings, age gaps between children, the social contexts of individuals, stereotypes about birth order, and the culture of the individuals (Keller \& Zach, 2002; Carlson, Watts, \& Maniacci, 2006; Zajonc, 2001; Herrara et al., 2003; Horner et al, 2012). 
Adler's theory included a section explaining that the genders of children can impact their psychological birth order and their parents' treatment of their children. For example, he posited that if a boy were the second born child behind a girl, he may take on the role of the first born. He also mentioned that in a family of multiple children of entirely the same gender except for one, the child of the opposite gender may develop a slightly different personality than would be expected based on birth rank alone (Ryckman, 2013). While the research in this area is limited, Keller and Zach (2002) conducted a study focusing on how gender and birth order served as determinants of parental behavior.

The study focused on male and female infants of varying birth ranks from a northern German area and how mothers and fathers treated their children differently based on both gender and birth order. Previous research has shown that first born babies receive significantly more parental attention in terms of interaction time and feedings than later born children (Keller \& Zach, 2002). This is consistent with the idea that first born children initially have no competition for parental attention and therefore receive more of it than their subsequent siblings. Keller and Zach (2002) also found that gender impacts how parents treat their offspring. Their data supported the same sex hypothesis, which states that parents prefer their children who have the same gender as they do. Therefore, their results showed that mothers preferred their daughters in terms of care and presence, while fathers preferred their sons in terms of presence. When both parents were present, they preferred first born males, which likely reflects the impact of a patriarchal society on how children are raised. Overall, the study showed that both gender and birth order impact parental behavior. Adler stated that it was not necessarily one's birth rank that impacts development, but the situation into which a child is born. Since the gender of children has been found to impact the way they are raised, it would therefore influence the environment in which children grow up. Thus, gender can play a role in birth order effects due to how parents treat their children based on their sex and birth rank.

Adler also wrote that psychological birth order may be different from actual birth order depending on the age gaps between subsequent offspring (Ansbacher \& Ansbacher, 1956). Carlson, Watts, and Maniacci (2006) found that age differences of five years or greater often separate children into distinct subgroups that alter the impact of actual birth order positions (Eckstein et al., 2010). They also suggest that this may be because children typically begin school at age five and would therefore have limited contact with newborn siblings (Eckstein et al., 2010). Zajonc (2001) also found that age gaps could be relevant in terms of the relationships between siblings. An important interaction between siblings is the teaching and modeling relationship that develops between older and younger siblings. However, these tutorial functions do not develop until two or three years after a new sibling is born. This may impact interactions between siblings in large families. If the first born child is significantly older than a newborn child, it will be another few years before the birth order effects between siblings begin to develop. If the child is much older, the interactions between them may be limited and their relationships may be between themselves and the siblings who are closer to their own age. Therefore, there may be two separate groups of siblings within one family who adopt birth order positions among those groups. This shows that Adler's theory that age gaps may lead to a change in perceived birth order positions and altered characteristics is supported by research.

While some of the factors that can impact birth order effects were part of Adler's initial theory, others have led researchers to question the validity of his work. Harris (2000) contests that birth order effects are only relevant in certain contexts. She writes that in order for behavior to occur in multiple contexts, there must be past evidence that their behavior will be helpful in various situations. For example, children may act a certain way with their friends, but act in an entirely different way around their parents because that behavior would not be appropriate in both contexts. Consequently, Harris (2000) believes that birth order effects are only present within the familial context and are not relevant outside the family. She also cites research studies that have been conducted on the birth order theory that did not show support for Adler's work, finding that all individuals self-report having certain characteristics regardless of birth rank. She contests that there is little validity to Adler's theory as children age and develop lives outside of their family context.

The stereotypes of the characteristics that develop as a result of one's birth rank are another factor that may influence the effects of birth order on personality. Herrara et al. (2003) analyzed four studies relating to beliefs about birth rank and their reflection in reality. Three of 
the studies examined the stereotypes that have developed about birth order. They found that people believe birth order leads to the development of distinct personalities, that higher birth ranks are likely to attain more occupational prestige, and that the personality traits commonly attributed to older siblings favor the attainment of higher occupational prestige. Therefore, the first three studies focused on identifying the stereotypes about birth order which were consistent with both Adler's theory and the actual traits research has found to be present in individuals.

The fourth study focused on the actual occupational prestige and level of academic achievement of birth ranks, finding that older siblings do attain more prestigious occupations and complete more years of schooling (Herrara et al, 2003). Thus, the study found that there is empirical support to corroborate the stereotypes behind birth order. The work of Herrera et al. (2003) is very comprehensive because it both establishes the beliefs of the population and also determines that people do embody these stereotypes. However, it is unclear whether individuals have these characteristics as a result of birth order or as a result of society's expectations for individuals of each birth rank. It is possible that because oldest children are expected to be very high achieving and have prestigious careers, they fit themselves into those roles. This means that society's beliefs about birth order may factor into the development of specific characteristics.

A final factor that may impact birth order effects is the culture of the individuals. The vast majority of the studies conducted on the topic were composed of European and North American participants (Eckstein et al., 2010). There is a significant lack of research that centers on individuals representing other cultures. It is possible that the birth order theory only applies to certain cultures, but more research is needed to determine whether the results of previous studies can be generalized to all people. For example, the finding that first born sons tend to be preferred and go on to hold political positions may be a reflection of the ideals of a patriarchal society. Horner et al. (2012) conducted a study among youths in Latin America and determined that first born children tend to have higher achievement, that first born sons tend to be favored, and that younger children are more at-risk for substance use. These findings are consistent with Adler's theory of birth order, but it is unclear why individuals fit these descriptions. It is possible that a lack of wealth contributes to the relative success of the first born children. If the family has a limited amount of money, they may spend it on their first child's education. The differences between siblings could also be due to variances in parental attention, which would be consistent with the findings in other societies. Because the cultures of different people vary so significantly and there is limited research on its relation to birth order, it is possible that culture impacts the effects of birth rank on personality development.

\section{Discussion of Research}

The research conducted over the years on Adler's theory of birth order and its possible effects on personality development seems to be comprehensive. However, while there is a fair amount of research in that area, it also has flaws and limitations. One of the most significant flaws of the previously conducted birth order studies is that they failed to distinguish between actual and psychological birth order. Actual birth order refers to the successive number of births of siblings, whereas psychological or perceived birth order refers to one's perception of his or her birth order, which may be different from actual birth rank. Adler emphasized in all of his work that perceived birth order is more important than the successive number of births. He felt that it was the experiences individuals have because of their position within the family that impacts their development, rather than just the rank itself (Ansbacher \& Ansbacher, 1956). However, it can be difficult for researchers to determine perceived birth order, so they typically rely on actual birth order positions to classify participants in their studies. This may impact the results because some participants' psychological birth ranks may be different from their actual positions. Therefore, they would be placed into a category that does not align with how they perceive themselves. Their traits would be included in those for each birth order position and could skew the results. However, it is unclear how often actual and psychological birth order differ, so it is difficult to determine how flawed studies using actual birth order may be. In studies that actually do use perceived birth order to classify participants, there is a risk of human error altering the results. These studies rely on individuals determining their own birth order. Whenever people are allowed to evaluate themselves, there is the risk that they will misrepresent themselves, whether intentionally or 
not. They may be aware of the stereotypes regarding each birth order position and lie about their perceived position or simply view themselves as having the role of a particular rank when this is actually not the case. It is also possible that perceived birth order may change over time and the way individuals perceive themselves at any given time may vary. Therefore, studies using psychological birth order may also be flawed. The majority of previous research was composed of participants who were classified based on their actual birth order. Thus, that research may be slightly inaccurate depending on how drastically and how often perceived and actual birth order are different.

Another potential limitation of the research is that Adler distinguished between a second born child and a middle child. Middle children have only one younger and one older sibling, whereas second born children have at least two younger siblings. However, the research studies did not classify them differently and grouped all children who were neither the youngest nor oldest into the middle child category. This likely reflects the difference between the time periods in which Adler developed his theory and when the research was conducted. During Adler's time, families were likely to have more children than during recent years. Therefore, for research studies conducted more recently, it may have been difficult to find enough people who fit the second born position to include them in the study. Even if they were found, they may consider themselves to be a middle born child because the distinction between a second and middle child has diminished over time. Because of this, it is unlikely that the loss of the second born group has really impacted more recent studies, although that is a potential flaw in rare cases.

The use of self-report and correlation procedures is another potential limitation of birth order research. It is very important to note that correlation does not determine causation, so it is possible that the correlations between personality traits and birth order may be due to chance. However, it seems unlikely that this is the case because of the great number of studies that have shown support for Adler's theory. The few studies whose results did not show a connection between birth order and personality relied entirely on self-report measures. People are known for misrepresenting themselves, both intentionally and unintentionally. The odds of people responding inaccurately to survey questions relating to personality traits are increased because of the nature of the questions. They may be biased in their own self concepts and may not want to admit to having certain traits. Therefore, studies utilizing self-report measures are more likely to be flawed. However, it would be difficult to design a birth order study that does not rely at all on these methods, so it is important that researchers work to design surveys and questionnaires in ways that diminish flaws due to participants being able to lie or omit information.

A final major limitation of the birth order research is the lack of diversity among those who have been studied. Almost all of the studies have focused on European and North American individuals, which means that the majority of the research has focused on Caucasians (Eckstein et al, 2010). Because of this, the research cannot reliably be generalized to other populations, ethnicities, and cultures. There is some literature on other cultures, including Latin youth, as previously discussed, that has shown support for Adler's work in other cultures. Therefore, while the results of studies cannot be generalized to all populations, it is possible and perhaps even likely that the majority of people display birth order effects.

Overall, the research shows support for Adler's theory of how birth order positions can impact the development of personality traits. Reviews of hundreds of studies have shown support for the distinct personalities that may form as a result of birth rank (Eckstein et al, 2010). While some studies did not find any empirical evidence to corroborate the theory, they are few in number and all used measures that are not entirely reliable. There is enough empirical support to determine that birth order does impact the development of personality. Both the descriptions of the characteristics each birth order position is expected to have and the possible factors Adler believed could impact those effects have been corroborated through research studies. There is a significant need for more research to be conducted on birth order and personality, but the current data show a clear relationship between them.

\section{Suggestions for Future Research}

While prior research showed clear support for Adler's theory, more studies should be conducted to verify their results and account for the family structures that are more common for this time period. Adler's work is from the 1920 s, so the factors he wrote about that could impact the effects of birth order have changed and evolved 
over time. Because it was more common for children to die during that time period, he wrote about how the deaths of children could alter the perceived birth order positions of the remaining siblings (Ansbacher \& Ansbacher, 1956). However, it is less common for individuals to die during childhood today, so it is more likely that the psychological impact of a sibling's death would have more of an impact than the change in perceived birth order position. While the effect of the death of a sibling is less applicable today, the presence of a sibling with disabilities has become increasingly common. Adler did include this in his writings as a factor that could change one's perceived birth order position, but there is very little research in this area. However, autism continues to become more prevalent in today's society; more children are diagnosed with learning disabilities; and more psychiatric disorders are identified and treated in children. Therefore, there is an increased need for research studies on how having siblings with special needs impacts personality development. It is possible that a disability could alter perceived birth order positions, interactions between siblings in terms of teaching and modeling, and the process of de-identification. Studies should be conducted to determine how each of these factors is impacted by the special needs of individuals with disabilities.

Another factor that was not as relevant during Adler's time but should be analyzed is how birth order is relevant to nontraditional families. When the theory was created, most families consisted of a married man and woman and their children. However, it is increasingly common for children to be raised by same sex parents or single parents, be born out of wedlock, experience the divorce of their parents, and experience the remarriage of parents, which often includes the addition of new siblings to the family. Parental attention and affection has a significant role in the development of personality. It would be interesting to see how parents who are raising children without the help of a spouse or partner would impact this.

Adler also wrote during a time period when women typically did not work and spent most of their time raising their children. Since many women now have both careers and families, their attention must be divided between the two, and more children are placed in daycare facilities. The genders of parents and their children have also been shown to be relevant to birth order, so families with same sex parents should be studied. Blended fam- ilies are very common in society today, so their effects on birth order must be examined as well. When parents marry people other than their children's biological parents, children may effectively have more than just two parents and have step or half siblings. The addition of these new siblings would most likely impact how individuals perceive their position within their family, but significant research must be conducted in this area. It would also be interesting to study whether adopted children experience the same birth order impacts. If they are adopted later in life, they may have spent much of their childhood developing personalities without the presence of siblings. Their addition to families could alter both their own perception of themselves, as well as those of the children in their family. Therefore, all nontraditional family structures must be studied extensively in terms of parental attention and affection, the possible changes to perceived birth order positions, and how personality development is impacted as a result.

More research should also be conducted on other factors Adler wrote about that can impact birth order effects. In addition to studying the many factors that can lead to a change in an individual's birth order position, the likelihood of this happening should be studied. Since it is possible that previous research has been skewed because researchers chose to use actual birth order rather than perceived birth order, research should be conducted to determine how often people's birth order positions change. With this information, the validity of previous research could be determined more easily. There is also a lack of research on how age gaps between siblings can lead to the development of subgroups of siblings who take on the roles of different positions. The likelihood of this happening and how it can impact the personalities of those affected should be analyzed in more comprehensive research studies.

One of the most common criticisms of the current research on birth order is the populations that have been studied. The vast majority of research has focused on North American and Europeans and has shown support for Adler's theory (Eckstein et al, 2010). However, more recent and comprehensive research should be conducted to determine whether this holds true among other cultures and whether it is still true for the original populations that were studied. Researchers should start focusing on more diverse samples that include various ethnicities, races, and cultures across the globe to determine whether birth order is relevant to all people. If the 
research is expanded to include all populations, it can then be generalized to more people and Adler's theory could be more widely recognized and accepted.

A final suggestion for future research focuses on the prevalence of technology among children today. When Adler first developed his theory, the main social interactions for children took place between siblings. However, more children are placed in daycares and preschools when they are very young, which expands their social interactions to include many other children of the same age. They are also cared for by adults who are not their parents. Many children also grow up watching television shows and playing with iPads, which can help them learn. They are not as reliant on parents and siblings for learning and interactions because they are now so readily available through the use of technology. As children age, they may also utilize social networking sites, which greatly expands their social interactions to include people outside the familial context. New studies should be conducted on the interactions between siblings, teaching, and learning in terms of technology.

As time passes, society and what is considered normal among various cultures will inevitably change. Adler created his theory of birth order and its impact on personality development nearly one hundred years ago. Despite that, its effects have still been found through various studies in recent years. The research has shown a clear connection between birth order and personality. Because the factors that may impact birth order effects have evolved over time and new ones have developed, more updated research should be conducted. The need for more research does not detract from the validity of the theory since its necessity is inevitable to account for the changes that occur in society as time passes. Therefore, a thorough analysis of the comprehensive data shows that Adler's theory of how birth order impacts personality development is validated through many years of empirical research.

\section{References}

About Alfred Adler. History: About: Adler School of Professional Psychology. Retrieved from http://www.adler.edu/ page/about/history/about-alfred-adler

Ansbacher, H.L., \& Ansbacher, R. R. (Eds.). (1956). The individual psychology of Alfred Adler. New York: Basic Books.
Eckstein, D. (2000). Empirical studies indicating significant birth-order related personality differences. Journal of Individual Psychology, 56, 481-494.

Eckstein, D., Aycock, K.J., Sperber, M.A., McDonald, J., Van Wiesner III, V., Watts, R.E., \& Ginsburg, P. (2010). A review of 200 birth-order studies: lifestyle characteristics. Journal of Individual Psychology, 66, 408-434.

Eckstein, D., \& Kaufman, J.A. (2012). The role of birth order in personality: An enduring intellectual legacy of Alfred Adler. Journal of Individual Psychology, 68, 60-74.

Harris, J.R. (2000). Context-specific learning, personality, and birth order. Blackwell Publishers Inc., 9, 174-177.

Herrera, N.C., Zajonc, R.B., Wieczorkowska, G., \& Cichomski, B. (2003). Beliefs about birth rank and their reflection in reality. Journal of Personality and Social Psychology, 85, 142-150.

Horner, P.S., Andrade, F., Delva, J., Grogan-Kaylor, A., \& Castillo, M. (2012). The relationship of birth order and gender with academic standing and substance use among youth in Latin America. The Journal of Individual Psychology, 68, 19-37.

Keller, H., \& Zach, U. (2002). Gender and birth order as determinants of parental behavior. International Journal of Behavioral Development, 26, 177-184.

Recchia, H.E., Howe, N., \& Alexander, S. (2009). "You didn't teach me, you showed me": Variations in sibling teaching strategies in early and middle childhood. Merrill-Palmer Quarterly, 55, 55-75.

Ryckman, R.M. (2013). Theories of personality. California: Cengage Learning.

Stewart, A.E. (2012). Issues in birth order research methodology: Perspectives from individual psychology. Journal of Individual Psychology, 68, 75-106.

Zajonc, R.B. (2001). The family dynamics of intellectual development. American Psychologist, 56, 490-496. 